\title{
Acute and midterm outcomes of the post-approval MELODY Registry: a multicentre registry of transcatheter pulmonary valve implantation
}

Nordmeyer, Johannes ; Ewert, Peter ; Gewillig, Marc ; AlJufan, Mansour ; Carminati, Mario ; Kretschmar, Oliver ; Uebing, Anselm ; Dähnert, Ingo ; Röhle, Robert ; Schneider, Heike ; Witsenburg, Maarten ; Benson, Lee ; Gitter, Roland ; Bökenkamp, Regina ; Mahadevan, Vaikom ; Berger, Felix

\begin{abstract}
AIMS The post-approval MELODY Registry aimed to obtain multicentre registry data after transcatheter pulmonary valve implantation (TPVI) with the Melody ${ }^{\mathrm{TM}}$ valve (Medtronic plc.) in a largescale cohort of patients with congenital heart disease (CHD). METHODS AND RESULTS Retrospective analysis of multicentre registry data after TPVI with the Melody ${ }^{\mathrm{TM}}$ valve. Eight hundred and forty-five patients (mean age: $21.0 \pm 11.1$ years) underwent TPVI in 42 centres between December 2006 and September 2013 and were followed-up for a median of 5.9 years (range: 0-11.0 years). The composite endpoint of TPVI-related events during follow-up (i.e. death, reoperation, or reintervention $>48 \mathrm{~h}$ after TPVI) showed an incidence rate of $4.2 \%$ per person per year [95\% confidence interval (CI) 3.7-4.9]. Transcatheter pulmonary valve implantation infective endocarditis (I.E.) showed an incidence rate of $2.3 \%$ per person per year (95\% CI 1.9-2.8) and resulted in significant morbidity and in nine deaths. In multivariable Cox proportional hazard models, the invasively measured residual right ventricle (RV)-topulmonary artery (PA) pressure gradient (per $5 \mathrm{mmHg}$ ) was associated with the risk of the composite endpoint (adjusted hazard ratio: 1.21, 95\% CI 1.12-1.30; P < 0.0001) and the risk of TPVI I.E. (adjusted hazard ratio: $1.19,95 \%$ CI 1.07-1.32; $\mathrm{P}=0.002$ ). Major procedural complications (death, surgical, or interventional treatment requirement) occurred in $0.5 \%, 1.2 \%$, and $2.0 \%$, respectively. Acutely, the RVto-PA pressure gradient and the percentage of patients with pulmonary regurgitation grade $>2$ improved significantly from 36 [interquartile range (IQR) 24-47] to 12 (IQR 7-17) mmHg and 47 to 1\%, respectively $(\mathrm{P}<0.001$ for each). CONCLUSION The post-approval MELODY Registry confirms the efficacy of TPVI with the Melody ${ }^{\mathrm{TM}}$ valve in a large-scale cohort of CHD patients. The residual invasively measured RVto-PA pressure gradient may serve as a target for further improvement in the composite endpoint and TPVI I.E. However, TPVI I.E. remains a significant concern causing significant morbidity and mortality.
\end{abstract}

DOI: https://doi.org/10.1093/eurheartj/ehz201

Posted at the Zurich Open Repository and Archive, University of Zurich

ZORA URL: https://doi.org/10.5167/uzh-177407

Journal Article

Accepted Version

Originally published at:

Nordmeyer, Johannes; Ewert, Peter; Gewillig, Marc; AlJufan, Mansour; Carminati, Mario; Kretschmar, Oliver; Uebing, Anselm; Dähnert, Ingo; Röhle, Robert; Schneider, Heike; Witsenburg, Maarten; Benson, Lee; Gitter, Roland; Bökenkamp, Regina; Mahadevan, Vaikom; Berger, Felix (2019). Acute and midterm 
outcomes of the post-approval MELODY Registry: a multicentre registry of transcatheter pulmonary valve implantation. European Heart Journal, 40(27):2255-2264.

DOI: https://doi.org/10.1093/eurheartj/ehz201 
EURHEARTJ-D-17-01733 - R2

\title{
Acute and midterm outcomes of the post-approval MELODY Registry - a multicentre registry of transcatheter pulmonary valve implantation
}

\author{
(Brief title: MELODY Registry)
}

Johannes Nordmeyer ${ }^{1}$, Peter Ewert ${ }^{4,5,6}$, Marc Gewillig ${ }^{7}$, Mansour AlJufan ${ }^{8}$, Mario Carminati ${ }^{9}$, Oliver Kretschmar ${ }^{10}$, Anselm Uebing ${ }^{11}$, Ingo Dähnert ${ }^{12}$, Robert Röhle ${ }^{13}$, Heike Schneider ${ }^{14}$, Maarten Witsenburg ${ }^{15}$, Lee Benson ${ }^{16}$, Roland Gitter $^{17}$, Regina Bökenkamp ${ }^{18}$, Vaikom Mahadevan $^{19}$, and Felix Berger ${ }^{1,2,3}$, on behalf of the MELODY Registry investigators

\author{
German Heart Institute Berlin, Berlin, Germany ${ }^{1}$ \\ Charité University Medicine Berlin, Berlin, Germany²
}

DZHK (German Centre for Cardiovascular Research), Partner Site Berlin, Berlin, Germany ${ }^{3}$

German Heart Center Munich, Munich, Germany ${ }^{4}$

TUM School of Medicine, Technical University of Munich, Munich, Germany ${ }^{5}$

Munich Heart Alliance, Partner Site of the DZHK (German Centre for Cardiovascular Research), Germany ${ }^{6}$

Fetal and Pediatric Cardiology, University Hospitals Leuven, Belgium ${ }^{7}$

King Faisal Specialist Hospital and Research Centre, Heart Centre, Riyadh, Kingdom of Saudi Arabia ${ }^{8}$

Department of Pediatric Cardiology and Adult Congenital Heart Disease, San Donato Milanese (MI), Italy ${ }^{9}$

Department of Paediatric Cardiology, University Children's Hospital, Zurich, Switzerland ${ }^{10}$

Adult Congenital Heart Disease Centre, Royal Brompton Hospital, London, UK $^{11}$

Department of Pediatric Cardiology, University of Leipzig - Heart Centre, Leipzig, Germany ${ }^{12}$

Coordination Center of Clinical Studies, KKS, Charité University Medicine Berlin, Berlin, Germany ${ }^{13}$

Department of Pediatric Cardiology, University Medical Centre, Göttingen, Germany ${ }^{14}$

Department of Paediatric Cardiology, Erasmus University Medical Centre, Rotterdam, Netherlands ${ }^{15}$

Division of Cardiology, Department of Pediatrics, The Hospital for Sick Children, Toronto, Canada ${ }^{16}$

Department of Pediatric Cardiology, Kepler University Hospital, Linz, Austria ${ }^{17}$

Department of Pediatric Cardiology, Leiden University Medical Centre, Leiden, Netherlands ${ }^{18}$

Central Manchester University Hospitals, Manchester Royal Infirmary, Manchester, UK ${ }^{19}$ 


\section{Corresponding Author:}

Dr Johannes Nordmeyer

Department of Congenital Heart Disease - Pediatric Cardiology

Deutsches Herzzentrum Berlin

Augustenburger Platz 1

Tel: $\quad+493045932800$

Fax: $\quad+493045932900$

Email: nordmeyer@dhzb.de

Total word count: 6,459 words 


\begin{abstract}
Aims - The post-approval MELODY Registry aimed to obtain multicentre registry data after transcatheter pulmonary valve implantation (TPVI) with the Melody ${ }^{\mathrm{TM}}$ valve (Medtronic plc.) in a large-scale cohort of patients with congenital heart disease (CHD).
\end{abstract}

Methods and results - Retrospective analysis of multicentre registry data after TPVI with the Melody $^{\mathrm{TM}}$ valve. 845 patients (mean age: $21.0 \pm 11.1$ years) underwent TPVI in 42 centres between 12/2006 and 09/2013 and were followed-up for a median of 5.9 years (range: 0 to 11.0 years). The composite endpoint of TPVI-related events during follow-up (i.e. death, reoperation or reintervention $>48 \mathrm{~h}$ after TPVI) showed an incidence rate of $4.2 \%$ per person per year $(95 \%$ CI 3.7-4.9). TPVI infective endocarditis showed an incidence rate of $2.3 \%$ per person per year (95\% CI 1.9-2.8) and resulted in significant morbidity and in 9 deaths. In multivariable Cox proportional hazard models, the invasively measured residual RV-to-PA pressure gradient (per $5 \mathrm{mmHg}$ ) was associated with the risk of the composite endpoint (adjusted hazard ratio: 1.21, 95\% CI 1.12-1.30; $P<0.0001$ ) and the risk of TPVI infective endocarditis (adjusted hazard ratio: $1.19,95 \%$ CI $1.07-1.32 ; P=0.002$ ). Major procedural complications (death, surgical or interventional treatment requirement) occurred in $0.5,1.2$, and $2.0 \%$, respectively. Acutely, the right ventricle (RV)-to-pulmonary artery $(\mathrm{PA})$ pressure gradient and the percentage of patients with pulmonary regurgitation grade $>2$ improved significantly from 36 (interquartile range 2447) to 12 (interquartile range 7-17) $\mathrm{mmHg}$ and 47 to $1 \%$, respectively ( $P<0.001$ for each).

Conclusions - The post-approval MELODY Registry confirms the efficacy of TPVI with the Melody ${ }^{\mathrm{TM}}$ valve in a large-scale cohort of CHD patients. The residual invasively measured RVto-PA pressure gradient may serve as a target for further improvement in the composite endpoint and TPVI infective endocarditis. However, TPVI infective endocarditis remains a significant concern causing significant morbidity and mortality. 
Key Words: Congenital heart disease $\bullet$ Transcatheter pulmonary valve implantation $\bullet$ RVOT dysfunction 


\section{Introduction}

Transcatheter pulmonary valve implantation (TPVI) plays an important role within current treatment strategies for patients with congenital heart disease (CHD) and right ventricular outflow tract (RVOT) dysfunction ${ }^{1-9}$. RVOT dysfunction frequently occurs in these patients because of the limited lifespan of the material that was used during surgical repair.

Currently, two balloon-expandable devices are available for TPVI: the Melody ${ }^{\mathrm{TM}}$ valve (Medtronic plc, Ireland; CE-mark approval in 2006) and the SAPIEN XT (Edwards Lifesciences LLC, USA; FDA-approval in 2017). The devices have different specifications related to the intended TPVI diameters (Melody valve ${ }^{\mathrm{TM}}$ : 18, 20 and $22 \mathrm{~mm}$; SAPIEN XT: 20, 23, 26 and $29 \mathrm{~mm})$.

The post-approval MELODY Registry aimed to obtain multicentre registry data after transcatheter pulmonary valve implantation (TPVI) with the Melody ${ }^{\mathrm{TM}}$ valve in a large-scale cohort of patients with congenital heart disease (CHD). 


\section{Methods}

\section{Study design}

The post-approval MELODY Registry is an investigator-initiated, multicentre registry after TPVI with the Melody ${ }^{\mathrm{TM}}$ valve outside the United States of America. Participating centres were approached during Melody ${ }^{\mathrm{TM}}$ valve implanters meetings in Europe, regardless of the implanting physician's level of past experience with TPVI. This strategy was adopted to achieve a large number of included patients and to allow for an assessment of observational data obtained in routine clinical practice. Overall, 42 cardiac centres contributed data of patients who received TPVI with the Melody ${ }^{\mathrm{TM}}$ valve after CE-mark approval.

In this study, patients were registered retrospectively. Firstly, study centres submitted anonymized data on patients who underwent TPVI with the Melody ${ }^{\mathrm{TM}}$ valve in the pulmonary position during the study period $(\mathrm{n}=1,080)$. Secondly, during computerized data management that was performed by the Coordination Center of Clinical Studies (KKS, Charité University Medicine Berlin), specified exclusion criteria were applied to allow for a consistent dataset that would be amenable for further statistical analysis (Supplemental Figure 1). Thus, 845/1,080 datasets $(78.2 \%)$ were included for analysis.

The study adhered to international rules for scientific studies and the Helsinki principles. Consent was given by patients and/or parents/guardians as appropriate. The institutional review board or ethics committee at participating centres approved the submission of data. The events and values collected were site reported, and there were no core laboratories. The coordinating centre of the MELODY Registry was the German Heart Institute Berlin, Germany, where the data were collected and analyzed. The decision to undergo TPVI with the Melody ${ }^{\mathrm{TM}}$ valve was made at each participating centre's discretion reflecting international guidelines ${ }^{14}$. 
General inclusion and exclusion criteria for TPVI with the Melody ${ }^{\mathrm{TM}}$ valve as well as details of the implantation procedure have been described previously ${ }^{1-9}$.

\section{Endpoints}

Primary endpoints of the study included a composite endpoint of TPVI-related follow-up events (i.e. death, reoperation or reintervention $>48 \mathrm{~h}$ after TPVI) and TPVI infective endocarditis according to modified Duke criteria (possible or definite) ${ }^{10-13}$.

Secondary endpoints of the study included procedural complications of TPVI with the Melody $^{\mathrm{TM}}$ valve (i.e. any adverse event that occurred during the procedure or $\leq 48 \mathrm{~h}$ after TPVI), invasive assessment of acute hemodynamic changes after TPVI with the Melody ${ }^{\mathrm{TM}}$ valve and non-invasive follow-up assessment up to 10-14 months after TPVI (labelled '1year'). Noninvasive follow-up assessment was performed according to each institution's routine practice and not in a protocol-specified manner; no dates of assessment were recorded. Usually, patients were assessed within 1-3 months post-TPVI (labelled 'post') and within 10-14 months after TPVI (labelled '1year'). The exam techniques that were applied during the non-invasive follow-up assessment included echocardiography, clinical assessment (NYHA-class) and chest $\mathrm{X}$-ray investigation; more detailed information on the non-invasive follow-up assessment is provided in the Supplemental Figure Legends. Presence of TPVI stent fractures were graded according to the stent fracture classification by Nordmeyer et al., with the known limitations of fracture identification within multiple stent frames ${ }^{15}$.

Data on the invasive assessment of acute hemodynamic changes was available for $635 / 845$ (75\%) to $826 / 845$ (98\%) patients (Table 6); data on the non-invasive follow-up assessment was available for $413 / 845$ (49\%) to 781/845 (92\%) patients (Supplemental Figure 4). Information on the presence of stent fractures at '1-year' was available in 633/845 (75\%) patients. 


\section{Statistics}

Continuous data are expressed as mean $\pm \mathrm{SD}$ or median with interquartile range (IQR) or range while categorical data are shown as absolute and relative frequencies (percentages). Continuous data were compared using unpaired Student's $t$ tests or with Mann-Whitney U tests where appropriate. Kruskal-Wallis-test was applied to compare more than two groups. Categorical variables were compared using the $\chi^{2}$ test or a Fisher's exact test if the expected cell frequencies were $<5$. For procedural complications, 95\%-Confidence intervals $(\mathrm{CI})$ of the observed relative frequencies were calculated using the method of Agresti-Coul1 ${ }^{16}$. Wilcoxon signed rank tests were performed to compare dependent data. For acute hemodynamic outcomes, 95\%-CIs of the median of the differences between pre and post measurements were calculated using the 'Bias corrected and accelerated method' or the 'bootstrap percentile

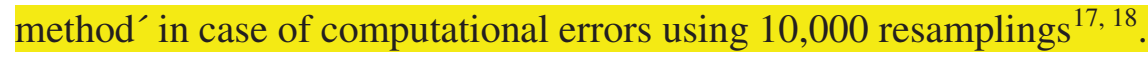

Cumulative incidence functions were created to display the time-to-first event of the composite endpoint (i.e. TPVI-related death, reoperation and reintervention) and of TPVI infective endocarditis accounting for 'non-TPVI related death' or 'non-TPVI right ventricular outflow tract obstruction (RVOTO) ${ }^{\prime}$ as competing risks. Starting point for the follow-up time was the day of intervention and follow-up times were summarized using median and range. Patients who underwent a TPVI-related reintervention with balloon dilation due to other reasons than TPVI infective endocarditis remained under follow-up for the assessment of TPVI infective endocarditis. For time-to-event outcomes, multivariable Cox proportional hazards models were created to calculate cause-specific hazards, and the assumptions of proportional hazards as well as goodness-of-fit were verified. Covariates for the multivariable models included calendar year of intervention, patient age and gender, primary hemodynamic lesion, pre-stenting, size of delivery system, and the invasively measured residual RV-to-PA pressure gradient (per 5 $\mathrm{mmHg}$ ). Further, linear regression was done to examine the relationship between original conduit size and the intended size of the delivered TPVI (i.e. size of the Ensemble ${ }^{\mathrm{TM}}$ delivery 
system). For all tests a $P$-value of $<0.05$ was considered statistically significant although results are to be considered as exploratory. Statistical analysis was performed on the SAS software (Version 9.4; SAS Institute Inc., Cary, NC, USA), R (Version 3.5.0 including the packages survival, survminer, and survMisc), and GraphPad Prism 5 (GraphPad Software, San Diego, CA, USA). 


\section{Results}

\section{Patient characteristics}

Baseline data are presented in Table 1. Between December 2006 and September 2013, 845 patients underwent TPVI with the Melody ${ }^{\mathrm{TM}}$ valve (Supplemental Figure 1) and were followed-up for a median of 5.9 years (range 0 days to 11 years). Patients were enrolled from 42 centres (Figure 1), of which 27 centres enrolled $<20$ patients and 15 centres $\geq 20$.

\section{Procedural details}

Details on the procedural variables are summarized in Table 2. Pre-stenting with at least one bare metal stent prior to TPVI was performed in the majority of patients $(737 / 845,87 \%)$. In patients who underwent TPVI with the Melody ${ }^{\mathrm{TM}}$ valve between the years 2006 and 2009 $(n=363)$, pre-stenting was used in 272/363 $(74.9 \%)$ patients, whilst in the more current years (2010-2013; $\mathrm{n}=482)$, pre-stenting was used in 465/482 (96.5\%) patients $(P<0.001)$. On average, the pre-stenting procedure added 28 minutes to the overall procedure time and 5 minutes to the overall fluoroscopy time when compared to patients who underwent TPVI only (Supplemental Tables 1 and 2). Over half Melody ${ }^{\mathrm{TM}}$ valves were implanted on a 22-mm Ensemble ${ }^{\mathrm{TM}}$ delivery system $(452 / 845,53.5 \%)$. The relationship between the intended size of delivered TPVI (i.e. size of Ensemble ${ }^{\mathrm{TM}}$ delivery system) and the original conduit diameter is displayed in Supplemental Figure 3. 


\section{Primary endpoints}

\section{Composite endpoint}

The composite endpoint of TPVI-related death, reoperation and reintervention showed an incidence rate of $4.2 \%$ per person per year (95\% CI 3.7-4.9). The corresponding cumulative incidence plot is presented in Figure 2.

Tabulated information on the composite endpoint events can be found in Supplemental Table 4. A composite endpoint event occurred in 207/845 (24.5\%) patients during follow-up: there were 5/845 (0.6\%) deaths due to fulminant endocarditis, 80/845 (9.5\%) reoperations with TPVI explantation (TPVI infective endocarditis, $n=53$; stenosis of the Melody valve ${ }^{\mathrm{TM}}, \mathrm{n}=20$; major TPVI stent fractures, $\mathrm{n}=6$; chronic pericardial effusion, $\mathrm{n}=1$ ) and 122/845 (14.4\%) reinterventions (pulmonary re-stenosis, $\mathrm{n}=88$; major TPVI stent fracture, $\mathrm{n}=18$; TPVI infective endocarditis, $\mathrm{n}=16$ ), of which 72/122 (59\%) related to balloon dilation of TPVI and 50/122 (41\%) to repeat TPVI as 'valve-in-valve' procedures.

In a multivariable Cox proportional hazard model (Table 3), 4 covariates showed a statistically significant association to the composite endpoint of TPVI-related follow-up events: patient age (adjusted hazard ratio: $0.96,95 \%$ CI $0.95-0.98 ; P=0.0001$ ), patient gender: female (adjusted hazard ratio: $0.67,95 \%$ CI $0.49-0.93 ; P=0.015)$, size of delivery system: $22-\mathrm{mm}$ (vs. $18-\mathrm{mm}$ ) (adjusted hazard ratio: $0.59,95 \%$ CI $0.41-0.86 ; P=0.006$ ) and residual RV-to-PA pressure gradient (per $5 \mathrm{mmHg}$ ) (adjusted hazard ratio: 1.21, 95\% CI 1.12-1.30; $P<0.0001$ ). 


\section{TPVI infective endocarditis}

TPVI infective endocarditis (I.E.) showed an incidence rate of $2.3 \%$ per person per year $(95 \%$ CI 1.9-2.8). The corresponding cumulative incidence plot is presented in Figure 3.

In the MELODY Registry, 109/845 (12.9\%) patients had TPVI I.E.; of those, 68/109 (62\%) were classified as definite and 41/109 (38\%) as possible TPVI I.E. according to modified Duke criteria. In 100/109 (92\%) patients, TPVI I.E. was blood-culture positive, whilst in 9/109 (8\%) patients, TPVI I.E. was blood-culture negative. In the majority of patients with blood-culture positive TPVI I.E., Staphylococcal species were found ( $\mathrm{n}=46 / 100,46 \%$; Supplemental Table 5).

Details on the primary management of TPVI I.E. and secondary outcomes are displayed in Supplemental Figure 5. Primary management of TPVI I.E. included conservative management with medical therapy in $41 / 109$ (38\%) patients, reintervention and medical therapy in $15 / 109$ (14\%) patients and reoperation with TPVI explantation and medical therapy in 53/109 (48\%) patients. However, 9/109 (8\%) patients died due to TPVI I.E. after conservative management $(n=5)$, reintervention $(n=2)$ and reoperation with TPVI explantation $(n=2)$, respectively.

The proportion of patients who underwent conservative therapy only was lower in patients with definite TPVI I.E. compared to those with possible TPVI I.E. according to modified Duke criteria $(\mathrm{n}=17 / 68,25 \%$ versus $\mathrm{n}=19 / 41,46 \% ; P=0.037)$. Furthermore, the proportion of patients who presented with hemodynamic instability or septic syndrome was higher in patients with definite TPVI I.E. compared to those with possible TPVI I.E. ( $\mathrm{n}=25 / 68,37 \%$ versus $\mathrm{n}=3 / 41,7 \% ; P=0.002)$. Further information on the secondary outcomes in the subgroups of patients with possible and definite TPVI I.E. can be found in Supplemental Table 6.

In a multivariable Cox proportional hazard model (Table 4), one covariate showed a statistically significant association to the composite endpoint of TPVI infective endocarditis: residual RV-to-PA pressure gradient (per $5 \mathrm{mmHg}$ ) (adjusted hazard ratio: 1.19, 95\% CI 1.07$1.32 ; P=0.002)$. 


\section{$\underline{\text { Secondary endpoints }}$}

\section{Procedural complications}

Details on procedural complications are summarized in Table 5. Four patients $(0.5 \%)$ died due to procedural complications (coronary compression, $\mathrm{n}=3$; fatal bleeding, $\mathrm{n}=1$ ). All other patients survived until discharge. Surgical or interventional treatment of a procedural complication was necessary in $10(1.2 \%)$, and in 17 patients $(2.0 \%)$, respectively. In 50 patients $(5.9 \%)$, procedural complications could be managed conservatively.

\section{Invasive assessment of acute hemodynamic changes after TPVI}

Acutely, TPVI led to a significant reduction of the invasively measured peak RV-to-PA pressure gradient from 36 (IQR 24-47) to 12 (IQR 7-17) $\mathrm{mmHg}(P<0.001)$. Subsequently, there was a significant reduction in the RV systolic pressure from 61 (IQR 50-73) to 40 (IQR 34-49) $\mathrm{mmHg}$ and in the RV-to-systemic pressure ratio from 0.64 (IQR 0.5-0.8) to 0.37 (IQR 0.3-0.5) $(P<0.001$ for each). The PA diastolic pressure increased after TVPI from 10 (IQR 813) to 13 (IQR 10-16) $\mathrm{mmHg}(P<0.001)$. The overall data on acute hemodynamic outcomes with invasive pressure measurement are displayed in Table 6.

Non-invasive follow-up assessment up to '1-year'

In a subset of patients, data on serial non-invasive follow-up assessment was available for review (Supplemental Figure 4). There was sustained echocardiographic outcome at the '1-year' assessment when compared to early post-intervention (peak RVOT velocity: $2.58 \pm$ 0.69 vs. $2.61 \pm 0.64 \mathrm{~m} / \mathrm{s}, P=0.001$; pulmonary regurgitation grade $>2: 1$ vs. $1 \%$; peak TR-jet velocity: $3.1 \pm 0.7$ vs. $3.0 \pm 0.6 \mathrm{~m} / \mathrm{s}, P=0.16$ ). Furthermore, the early improvement in NYHAclass after TPVI was sustained at the '1-year' assessment. TPVI stent fractures led to reoperation in 6/633 (1.0\%) patients (Supplemental Table 4). 


\section{Discussion}

The objective of the post-approval MELODY Registry was to obtain multicentre registry data after TPVI with the Melody ${ }^{\mathrm{TM}}$ valve in a large-scale cohort of CHD patients. Currently, $\sim 350$ patients undergo TPVI with the Melody ${ }^{\mathrm{TM}}$ valve in Europe per year. The MELODY Registry included 845 patients from 42 cardiac centres and, thus, represents the largest post-approval cohort of patients after TPVI with the Melody ${ }^{\mathrm{TM}}$ valve to date.

\section{Composite endpoint}

The composite endpoint of TPVI-related death, reoperation and reintervention showed an incidence rate of $4.2 \%$ per person per year $(95 \%$ CI $3.7-4.9)$. In a multivariable Cox proportional hazard model, there was a strong association between the composite endpoint and the residual invasively measured RV-to-PA pressure gradient (per $5 \mathrm{mmHg}$ ) at the time of TPVI (adjusted hazard ratio: $1.21,95 \%$ CI 1.12-1.30; $P<0.0001$ ). These data suggest that one potential factor to reduce the risk of the composite endpoint could be to reduce the residual RVto-PA pressure gradient at the time of TPVI. However, further research is necessary to assess the potential benefit of reduced residual RV-to-PA pressure gradients during follow-up.

When arguing for targeted gradient reduction during TPVI, however, the risks of coronary compression and conduit rupture related to more forceful TPVI expansion need to be considered. In the MELODY Registry, $26 \%$ of the stents that were used for pre-stenting were covered CP-stents (Supplemental Table 2). Furthermore, an increasing use of covered stents for pre-stenting is reported as an attempt to prevent from bleeding in case of conduit rupture during stent implantation. The increased use of covered pre-stents might have an influence on outcome parameters and needs to be evaluated in further studies. 
Another variable that showed a statistically significant association with the composite endpoint

on multivariable testing was the use of a $22-\mathrm{mm}$ Ensemble $^{\mathrm{TM}}$ delivery system (vs. 18-mm: adjusted hazard ratio: $0.59,95 \%$ CI $0.41-0.86 ; P=0.006)$. It could be hypothesized that in the MELODY Registry, 22-mm Ensemble ${ }^{\mathrm{TM}}$ delivery systems were chosen more often in patients with - in relative terms - smaller conduits, leading to a slight "overexpansion" of the RV-to-PA conduits (Supplemental Figure 3). However, further research is required to assess the potential benefits of this approach.

\section{TPVI infective endocarditis}

TPVI infective endocarditis (I.E.) showed an incidence rate of $2.3 \%$ per person per year (95\% CI 1.9-2.8). This data is comparable to a previous study with pooled data from 311 patients that showed an annualized I.E. rate of $2.4 \%$ after TPVI with the Melody ${ }^{\mathrm{TM}}$ valve ${ }^{11}$. The clinical impact of TPVI I.E. was substantial in the MELODY Registry since it was associated with significant morbidity and 9 follow-up deaths. In the MELODY Registry, 68/109 (62\%) patients were classified as definite and 41/109 (38\%) as possible TPVI I.E. according to modified Duke criteria ${ }^{10-13}$. Although in the MELODY Registry, patients with possible TPVI I.E. seemed to present with less severe disease compared to those who were classified as definite TPVI I.E., the clinical utility of the modified Duke criteria related to the diagnostic classification and prognostication of TPVI I.E. remains to be determined. In particular, there are known limitations related to the visualization of the Melody valve ${ }^{\mathrm{TM}}$ due to artifacts of the stent frame ${ }^{10,11}$, which might impact on the sensitivity and specificity of one of the major modified Duke criteria (i.e. 'imaging positive for I.E.'). Thus, a revised clinical classification system for TPVI I.E. and longer follow-up might be useful to study this clinically relevant follow-up complication. 
In the majority of patients with blood-culture positive TPVI I.E., Staphylococcal species were found ( $n=46 / 100,46 \%)$. TPVI I.E. with Staphylococcal species led to $7 / 9$ (78\%) of deaths that were attributed to TPVI I.E.. These findings are similar to previous findings that were obtained in an analysis of 76 unique published cases of endocarditis after TPVI with the Melody ${ }^{\mathrm{TM}}$ valve (Staphylococcal species: found in $47 \%$ cases, led to $8 / 9$ (89\%) deaths attributed to TPVI I.E.) $)^{13}$.

The findings of a multivariable Cox proportional hazard model suggest that one potential factor to reduce the risk of developing TPVI I.E. could be to reduce the residual RV-to-PA pressure gradient at the time of TPVI. However, further research is necessary to assess the potential benefit of reduced residual RV-to-PA pressure gradients. Because there is only limited knowledge about specific risk factors (e.g. discontinuation of antiplatelet therapy during follow-up) for the development of I.E. after $\mathrm{TPVI}^{10}$ as yet, patients should be strongly encouraged to apply general measures of endocarditis prevention (e.g. good dental hygiene). Furthermore, patients and medical personnel should be made aware of early signs of I.E. to allow for immediate treatment.

\section{Procedural complications}

Overall, major procedural complications (death, surgical or interventional treatment required) occurred in $4.2 \%$ in the MELODY Registry, which is comparable to previous studies including the expanded FDA Melody ${ }^{\mathrm{TM}}$ valve trial ${ }^{3,8}$. Coronary compression after TPVI ${ }^{19,20}$ occurred in five patients $(0.6 \%)$ leading to procedure-related death in three patients $(0.35 \%)$ and emergency surgery in two patients $(0.25 \%)$. Importantly, three patients after previous Ross-procedure suffered from coronary compression; these patients are already known to have a high risk for this major complication after $\mathrm{TPVI}^{20}$. Although the risk of coronary compression can be evaluated by thorough assessment prior to TPVI, it cannot be avoided completely. When highpressure pre-dilation of the intended implantation site is performed, there is a risk of conduit rupture that needs to be considered. In the MELODY Registry, the incidence of fatal conduit rupture $(\mathrm{n}=1,0.1 \%)$ was lower than the risk of fatal coronary compression $(\mathrm{n}=3,0.35 \%)$. 


\section{Hemodynamic outcome}

Acutely, TPVI with the Melody ${ }^{\mathrm{TM}}$ valve led to a significant reduction of the RV-to-PA pressure gradient and to a complete restoration of pulmonary valve function. These hemodynamic outcomes were sustained at '1-year' assessment, which is in line with previous studies $3,8,21,22$ confirming the hemodynamic efficacy of TPVI with the Melody ${ }^{\mathrm{TM}}$ valve.

\section{Limitations}

The most important limitations are the retrospective nature of the registry with self-reporting of data; furthermore, there was no auditing of data. Although the completeness of data on the primary endpoint parameters (i.e. composite endpoint and TPVI I.E.) was 100\%, there was limited completeness of data on the secondary endpoint parameters, particularly for the non-invasive follow-up assessment.

\section{Conclusions}

The post-approval MELODY Registry confirms the efficacy of TPVI with the Melody ${ }^{\mathrm{TM}}$ valve in a large-scale cohort of CHD patients. The composite endpoint of TPVI-related death, reoperation and reintervention showed an incidence rate of $4.2 \%$ per person per year $(95 \% \mathrm{CI}$ 3.7-4.9). The residual invasively measured RV-to-PA pressure gradient may serve as a target for further improvement in the composite endpoint and TPVI infective endocarditis (I.E.). However, TPVI I.E. remains a concern; TPVI I.E. showed an incidence rate of $2.3 \%$ per person per year (95\% CI 1.9-2.8) and resulted in significant morbidity and mortality; therefore, patients should be strongly encouraged to apply general measures of endocarditis prevention (e.g. good dental hygiene). Furthermore, patients and medical personnel should be made aware of early signs of I.E. to allow for immediate treatment. 


\section{Acknowledgements}

The following investigators and institutions participated in the MELODY Registry, listed in alphabetical order: Yacine Aggoun, Children's University Hospital of Geneva; Gabriella Agnoletti, Ospedale Infantile Regina Margherita; Helmut Baumgartner, University Hospital Muenster; Jose Suarez de Lezo, Reina Sofia Hospital Cordoba; Hugues Dessy, HUDERF Brussels; Daniel De Wolf, University Hospital of Ghent; Sven Dittrich, University Hospital Erlangen; Gaute Døhlen, Oslo University Hospital; Mathias Emmel, University Hospital of Cologne; Kristian Emmertsen, Aarhus University Hospital; Stephan Fichtlscherer, Goethe University Frankfurt; Harald Gabriel, Medical University of Vienna; Oliver Gaemperli, University Hospital Zurich; Andreas Gamillscheg, Medical University Graz; François Godart, CHU Lille; Patrice Guérin; CHU Nantes; Michael Hofbeck, University Children's Hospital Tuebingen; Gerard Martí, Hospital Vall d’Hebron; Ina Michel-Behnke, Medical University of Vienna; Ornella Milanesi, University of Padua; Fatima Pinto, University of Lisbon; Shakeel Qureshi, Evelina London Children's Hospital; Dietmar Schranz, University Hospital Giessen; Horst Sievert, CardioVascular Centre Frankfurt; Juha-Pekka Sinisalo, Helsinki University Hospital; Thierry Sluysmans, Cliniques Universitaires Saint-Luc; Isabella Spadoni, Fondazione Regione Toscana G. Monasterio.

\section{Conflicts of interest}

J.N. received honoraria from Medtronic plc. P.E., M.G., M.A.J., M.C., O.K., I.D., M.W., L.B., R.G., V.M. and F.B. received consultancy fees from Medtronic plc. 


\section{Funding}

The study was supported with an unrestricted research grant by Medtronic plc., Dublin, Ireland. Medtronic plc. had no role in designing the study, data acquisition or in the writing or approval of the manuscript. The unrestricted research grant was used to finance the external data management and statistical analysis, which was performed by the Charité Coordinating Center for Clinical Studies (KKS) located at the Charité University Medicine Berlin. Furthermore, the research grant was used to finance a study nurse. The authors aggregated end-point events, had unrestricted access to the data at the completion of the primary end-point analysis, reviewed the analysis with the study statistician, and wrote all versions of the manuscript. 


\section{References}

1. Bonhoeffer P, Boudjemline Y, Saliba Z, Merckx J, Aggoun Y, Bonnet D, Acar P, Le Bidois J, Sidi D, Kachaner J. Percutaneous replacement of pulmonary valve in a right-ventricle to pulmonary-artery prosthetic conduit with valve dysfunction. Lancet 2000;356(9239):1403-5.

2. Eicken A, Ewert P, Hager A, Peters B, Fratz S, Kuehne T, Busch R, Hess J, Berger F. Percutaneous pulmonary valve implantation: two-centre experience with more than 100 patients. Eur Heart J 2011.

3. McElhinney DB, Hellenbrand WE, Zahn EM, Jones TK, Cheatham JP, Lock JE, Vincent JA. Shortand medium-term outcomes after transcatheter pulmonary valve placement in the expanded multicenter US melody valve trial. Circulation 2010;122(5):507-16.

4. Zahn EM, Hellenbrand WE, Lock JE, McElhinney DB. Implantation of the melody transcatheter pulmonary valve in patients with a dysfunctional right ventricular outflow tract conduit early results from the u.s. Clinical trial. J Am Coll Cardiol 2009;54(18):1722-9.

5. Cools B, Brown SC, Heying R, Jansen K, Boshoff DE, Budts W, Gewillig M. Percutaneous pulmonary valve implantation for free pulmonary regurgitation following conduit-free surgery of the right ventricular outflow tract. Int J Cardiol 2015;186:129-35.

6. Butera G, Milanesi O, Spadoni I, Piazza L, Donti A, Ricci C, Agnoletti G, Pangrazi A, Chessa M, Carminati M. Melody transcatheter pulmonary valve implantation. Results from the registry of the Italian Society of Pediatric Cardiology. Catheter Cardiovasc Interv 2013;81(2):310-6.

7. Fraisse A, Aldebert P, Malekzadeh-Milani S, Thambo JB, Piechaud JF, Aucoururier P, Chatelier G, Bonnet D, Iserin L, Bonello B, Assaidi A, Kammache I, Boudjemline Y. Melody (R) transcatheter pulmonary valve implantation: results from a French registry. Arch Cardiovasc Dis 2014;107(11):607-14.

8. Lurz P, Coats L, Khambadkone S, Nordmeyer J, Boudjemline Y, Schievano S, Muthurangu V, Lee TY, Parenzan G, Derrick G, Cullen S, Walker F, Tsang V, Deanfield J, Taylor AM, Bonhoeffer P. Percutaneous pulmonary valve implantation: impact of evolving technology and learning curve on clinical outcome. Circulation 2008;117(15):1964-72.

9. Khambadkone S, Coats L, Taylor A, Boudjemline Y, Derrick G, Tsang V, Cooper J, Muthurangu V, Hegde SR, Razavi RS, Pellerin D, Deanfield J, Bonhoeffer P. Percutaneous pulmonary valve implantation in humans: results in 59 consecutive patients. Circulation 2005;112(8):1189-97.

10. McElhinney DB. Reflection and Rationalization: Making Sense of the Literature on Endocarditis After Transcatheter Pulmonary Valve Replacement. Circ Cardiovasc Interv 2017;10(2).

11. McElhinney DB, Benson LN, Eicken A, Kreutzer J, Padera RF, Zahn EM. Infective endocarditis after transcatheter pulmonary valve replacement using the melody valve: combined results of 3 prospective north american and European studies. Circ Cardiovasc Interv 2013;6(3):292-300.

12. Li JS, Sexton DJ, Mick N, Nettles R, Fowler VG, Jr., Ryan T, Bashore T, Corey GR. Proposed modifications to the Duke criteria for the diagnosis of infective endocarditis. Clin Infect Dis 2000;30(4):6338.

13. Habib G, Lancellotti P, Antunes MJ, Bongiorni MG, Casalta JP, Del Zotti F, Dulgheru R, El Khoury G, Erba PA, Iung B, Miro JM, Mulder BJ, Plonska-Gosciniak E, Price S, Roos-Hesselink J, Snygg-Martin U, Thuny F, Tornos Mas P, Vilacosta I, Zamorano JL, Erol C, Nihoyannopoulos P, Aboyans V, Agewall S, Athanassopoulos G, Aytekin S, Benzer W, Bueno H, Broekhuizen L, Carerj S, Cosyns B, De Backer J, De Bonis M, Dimopoulos K, Donal E, Drexel H, Flachskampf FA, Hall R, Halvorsen S, Hoen B, Kirchhof P, Lainscak M, Leite-Moreira AF, Lip GY, Mestres CA, Piepoli MF, Punjabi PP, Rapezzi C, Rosenhek R, Siebens K, Tamargo J, Walker DM. 2015 ESC Guidelines for the management of infective endocarditis: The Task Force for the Management of Infective Endocarditis of the European Society of Cardiology (ESC). Endorsed by: European Association for Cardio-Thoracic Surgery (EACTS), the European Association of Nuclear Medicine (EANM). Eur Heart J 2015;36(44):3075-128.

14. Baumgartner H, Bonhoeffer P, De Groot NM, de Haan F, Deanfield JE, Galie N, Gatzoulis MA, Gohlke-Baerwolf C, Kaemmerer H, Kilner P, Meijboom F, Mulder BJ, Oechslin E, Oliver JM, Serraf A, Szatmari A, Thaulow E, Vouhe PR, Walma E, Vahanian A, Auricchio A, Bax J, Ceconi C, Dean V, Filippatos G, Funck-Brentano C, Hobbs R, Kearney P, McDonagh T, Popescu BA, Reiner Z, Sechtem U, Sirnes PA, Tendera M, Vardas P, Widimsky P, Swan L, Andreotti F, Beghetti M, Borggrefe M, Bozio A, Brecker S, Budts W, Hess J, Hirsch R, Jondeau G, Kokkonen J, Kozelj M, Kucukoglu S, Laan M, Lionis C, Metreveli I, Moons P, Pieper PG, Pilossoff V, Popelova J, Price S, Roos-Hesselink J, Uva MS, Tornos P, Trindade PT, Ukkonen H, Walker H, Webb GD, Westby J. ESC Guidelines for the management of grown-up congenital heart disease (new version 2010): The Task Force on the Management of Grown-up Congenital Heart Disease of the European Society of Cardiology (ESC). Eur Heart J 2010. 
15. Nordmeyer J, Khambadkone S, Coats L, Schievano S, Lurz P, Parenzan G, Taylor AM, Lock JE, Bonhoeffer P. Risk stratification, systematic classification, and anticipatory management strategies for stent fracture after percutaneous pulmonary valve implantation. Circulation 2007;115(11):1392-7.

16. Agresti A. Approximate is better than 'exact' for interval estimation of binomial proportions. The American Statistician 1998(52):119-126.

17. Efron B. Better bootstrap confidence intervals. Journal of the American Statistical Association 1987(82):171-200.

18. Efron B. The Jackknife, the Bootstrap and other Resampling Plans. Society for Industrial and Applied Mathematics:Philadelphia 1982.

19. Sridharan S, Coats L, Khambadkone S, Taylor AM, Bonhoeffer P. Images in cardiovascular medicine. Transcatheter right ventricular outflow tract intervention: the risk to the coronary circulation. Circulation 2006;113(25):e934-5.

20. Morray BH, McElhinney DB, Cheatham JP, Zahn EM, Berman DP, Sullivan PM, Lock JE, Jones TK. Risk of coronary artery compression among patients referred for transcatheter pulmonary valve implantation: a multicenter experience. Circ Cardiovasc Interv 2013;6(5):535-42.

21. Cheatham JP, Hellenbrand WE, Zahn EM, Jones TK, Berman DP, Vincent JA, McElhinney DB. Clinical and hemodynamic outcomes up to 7 years after transcatheter pulmonary valve replacement in the US melody valve investigational device exemption trial. Circulation 2015;131(22):1960-70.

22. Hager A, Schubert S, Ewert P, Sondergaard L, Witsenburg M, Guccione P, Benson LN, Suarez de Lezo J, Lung TH, Hess J, Eicken A, Berger F. Five-year results from a prospective multicentre study of percutaneous pulmonary valve implantation demonstrate sustained removal of significant pulmonary regurgitation, improved right ventricular outflow tract obstruction and improved quality of life. EuroIntervention 2017;12(14):1715-1723. 


\section{Legends}

Figure 1. Geographical distribution of the study centres, including color-coding of the sample size per study centre.

Figure 2. Cumulative incidence plot of the composite endpoint of TPVI-related death, reoperation and reintervention. At 6 years the cumulative incidence of the composite endpoint was $20.8 \%$ (95\% CI 19.1-22.8), and at 8 years the cumulative incidence of the composite endpoint was $28.4 \%$ (95\% CI 25.6-31.4).

Figure 3. Cumulative incidence plot of TPVI infective endocarditis. At 6 years the cumulative incidence of TPVI infective endocarditis was $11.4 \%$ (95\% CI 10.3-12.5), and at 8 years the cumulative incidence of TPVI infective endocarditis was 16.2\% (95\% CI 14.6-18.1). 
Table 1. Baseline characteristics

Parameter

Overall population $(n=845)$

Age at TPVI (years), mean \pm SD

Sex (female / male), $n(\%)$

Height $(\mathrm{cm})$, mean \pm SD

Weight $(\mathrm{kg})$, mean $\pm \mathrm{SD}$

Primary hemodynamic lesion, $n(\%)$

Predominant pulmonary stenosis (PS) ${ }^{\mathrm{a}}$

$458 / 845(54)$

Predominant pulmonary regurgitation $(\mathrm{PR})^{\mathrm{b}}$

$182 / 845(22)$

Mixed lesion $^{\mathrm{c}}$

205/845 (24)

Principal diagnosis, $n(\%)$

Tetralogy of Fallot (TOF)

$263 / 845(31)$

Pulmonary atresia with VSD

$154 / 845(18)$

Truncus arteriosus communis

$92 / 845(11)$

TGA, pulmonary stenosis (PS), VSD

$80 / 845(9)$

Aortic valve disease, status post Ross-operation

$164 / 845(20)$

Double outlet right ventricle (DORV) with PS

$34 / 845$ (4)

Other / unknown

$58 / 845(7)$

Number of previous surgeries, median (range)

2 (0 to 6$)$

Right ventricular outflow tract (RVOT), $n(\%)$

Native or patch-extended RVOT

$46 / 845(6)$

Homograft conduit

$567 / 845(68)$

Heterograft conduit

92/845 (11)

Bioprosthetic heart valve (stented)

$61 / 845(7)$

Other / unknown

Original conduit diameter

Homograft or heterograft conduits, mean \pm SD (mm)

$$
20.3 \pm 3.2
$$

$$
\begin{aligned}
& \leq 18 \mathrm{~mm}, n(\%) \\
& 18-22 \mathrm{~mm}, n(\%) \\
& >22 \mathrm{~mm}, n(\%) \\
& \text { unknown, } n(\%)
\end{aligned}
$$$$
178 / 659 \text { (27) }
$$$$
271 / 659(41)
$$$$
142 / 659(22)
$$

Bioprosthetic heart valves (stented), mean $\pm \mathrm{SD}(\mathrm{mm})$ 
VSD, ventricular septal defect; TGA, transposition of the great arteries.

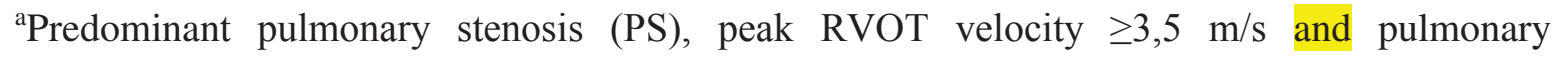
regurgitation < 'moderate' on echocardiography; ${ }^{b}$ predominant pulmonary regurgitation (PR), peak RVOT velocity $<3,5 \mathrm{~m} / \mathrm{s}$ and pulmonary regurgitation > 'moderate' on echocardiography; ${ }^{c}$ mixed lesion, patients who did not fit either the PS or PR categories. 
Table 2. Procedural variables

Procedural variables

Overall population $(n=845)$

Procedure time (min), mean \pm SD $152 \pm 56$

Fluoroscopy time $(\min )$, mean \pm SD $32 \pm 19$

Vascular access route, $n(\%)$

Trans-femoral

$805 / 845(95)$

Trans-jugular

$36 / 845(4.5)$

Other / unknown

Assessment of the relationship between coronary anatomy and RVOT, $n(\%)$

Performed prior to the TPVI catheterization procedure

$87 / 845(11)$

Performed during the TPVI catheterization procedure

Assessment with only one modality

Assessment with combinations of modalities

$413 / 758(54)$

Modalities used for assessment, $n(\%)$

Aortic root angiography

$642 / 758(85)$

Selective coronary angiography (SCA)

$392 / 758(52)$

SCA plus balloon inflation in RVOT

281/758 (37)

Balloon sizing of RVOT, $n(\%)$

High-pressure pre-dilation of RVOT, $n(\%)$

$405 / 845(48)$

Pre-Stenting prior to TPVI, $n(\%)$

No pre-stent

$108 / 845(13)$

1 pre-stent

$555 / 845(66)$

2 pre-stents

$148 / 845(17)$

3 pre-stents

$34 / 845(4)$

Size of Ensemble delivery system (EDS) for TPVI, $n(\%)$

$18-\mathrm{mm}$

$156 / 845$ (18)

20-mm

237/845 (28)

22-mm

452/845 (54)

High-pressure post-dilation of TPVI, $n(\%)$

540/845 (64)

Additional procedures during catheterization ${ }^{b}, n(\%)$

$129(15)$ 
RVOT, right ventricular outflow tract. ${ }^{a}$ It is of note that during high-pressure balloon dilation of RVOT and SCA, no data was recorded whether the high-pressure balloon was inflated to the predefined maximal diameter. ${ }^{b}$ Additional procedures during catheterization, e.g. balloon dilation or stenting of branch pulmonary arteries 
Table 3. Cox regression model related to the composite endpoint of TPVI-related death, reoperation and reinterve

\begin{tabular}{cccc} 
Variable & Coefficient & Standard error & $\begin{array}{c}\text { Hazard ratio } \\
\text { exp(coefficient) }\end{array}$ \\
& & & 0.91 \\
Patient age [years] & -0.10 & 0.05 & $\mathbf{0 . 9 6}$ \\
\hline Calendar year of TPVI procedure* & -0.04 & 0.01 & $\mathbf{0 . 6 7}$ \\
Patient gender: Female (vs. Male) & -0.40 & 0.16 & 1.16 \\
Lesion: Predominant PR (vs. predominant PS) & 0.15 & 0.20 & 0.95 \\
Lesion: Mixed (vs. predominant PS) & -0.05 & 0.18 & 0.73 \\
Prestenting: Yes (vs. No) & -0.31 & 0.19 & 0.75 \\
Size of delivery system: 20-mm (vs. 18-mm) & -0.29 & 0.20 & $\mathbf{0 . 5 9}$ \\
Size of delivery system: 22-mm (vs. 18-mm) & -0.52 & 0.19 & $\mathbf{1 . 2 1}$ \\
\hline Residual RV-to-PA pressure gradient [per 5 mmHg] & 0.19 & 0.04 & \\
\hline
\end{tabular}

*2006-2013. Significant differences in bold.

Test for proportional hazard assumption: $P=0.088$

Test for goodness-of-fit: $P=0.416$ 
Table 4. Cox regression model related to TPVI infective endocarditis

\begin{tabular}{cccc} 
Variable & Coefficient & Standard error & $\begin{array}{c}\text { Hazard ratio } \\
\text { exp(coefficient) }\end{array}$ \\
& & & \\
\hline Calendar year of TPVI procedure* & 0.09 & 0.07 & 1.10 \\
Patient age [years] & -0.01 & 0.01 & 0.99 \\
Patient gender: Female (vs. Male) & 0.03 & 0.21 & 1.03 \\
Lesion: Predominant PR (vs. predominant PS) & 0.20 & 0.26 & 1.22 \\
Lesion: Mixed (vs. predominant PS) & 0.01 & 0.24 & 1.01 \\
\hline Prestenting: Yes (vs. No) & 0.00 & 0.30 & 1.00 \\
Size of delivery system: 20-mm (vs. 18-mm) & -0.20 & 0.29 & 0.82 \\
Size of delivery system: 22-mm (vs. 18-mm) & -0.27 & 0.27 & 0.76 \\
Residual RV-to-PA pressure gradient [per 5 mmHg] & 0.17 & 0.05 & $\mathbf{1 . 1 9}$ \\
\hline
\end{tabular}

*2006-2013. Significant differences in bold.

Test for proportional hazard assumption: $P=0.189$

Test for goodness-of-fit: $P=0.597$ 


\begin{tabular}{l|c|cc}
\hline & $\begin{array}{c}\text { Overall number } \\
n(\% \text { of } n=845 ; 95 \% C l)\end{array}$ & $\begin{array}{c}\text { Conservative } \\
\text { management }\end{array}$ & $\begin{array}{c}\text { Interventional } \\
\text { treatment } \\
n(\% \text { of } n=845 ;\end{array}$ \\
\hline Coronary compression & $5(0.6 ; 0.21-1.42)$ & $0(0 ; 0-0.55)$ & $0(0 ; 0-0.55)$ \\
Bleeding due to conduit rupture & $7(0.8 ; 0.36-1.74)$ & $0(0 ; 0-0.55)$ & $6(0.7 ; 0.29-1.58)$ \\
Bleeding due to pulmonary artery & $5(0.6 ; 0.21-1.42)$ & $1(0.1 ; 0-0.74)$ & $3(0.4 ; 0.07-1.09)$ \\
Pre-stent dislodgment & $2(0.2 ; 0.01-0.92)$ & $1(0.1 ; 0-0.74)$ & $1(0.1 ; 0-0.74)$ \\
TPVI dislodgment & $3(0.4 ; 0.07-1.09)$ & $0(0 ; 0-0.55)$ & $1(0.1 ; 0-0.74)$ \\
Obstruction of pulmonary artery & $1(0.1 ; 0-0.74)$ & $0(0 ; 0-0.55)$ & $1(0.1 ; 0-0.74)$ \\
Balloon rupture & $13(1.6 ; 0.87-2.64)$ & $10(1.2 ; 0.61-2.20)$ & $3(0.4 ; 0.07-1.09)$ \\
Vascular access complications & $22(2.6 ; 1.70-3.93)$ & $18(2.1 ; 1.33-3.37)$ & $1(0.1 ; 0-0.74)$ \\
Arrhythmia & $15(1.8 ; 1.05-2.93)$ & $15(1.8 ; 1.05-2.93)$ & $0(0 ; 0-0.55)$ \\
Other ${ }^{\mathrm{a}}$ & $8(0.9 ; 0.45-1.89)$ & $5(0.6 ; 0.21-1.42)$ & $1(0.1 ; 0-0.74)$ \\
\hline Overall & $81(9.6 ; 7.77-11.77)$ & $50(5.9 ; 4.5-7.73)$ & $17(2.0 ; 1.24-3.22)$ \\
\hline
\end{tabular}

ather, e.g. dislocation of pacemaker / ICD-leads, injury of a right ventricular papillary muscle, pulmonary em confidence intervals (CI) correspond to an Agresti-Coull-Interval. 
Table 6. Acute hemodynamic outcome in the overall cohort, invasive pressure measurements

\begin{tabular}{|c|c|c|c|}
\hline & Pre & post & Median dift \\
\hline Parameter & \multicolumn{2}{|c|}{$\begin{array}{c}\text { median (IQR) } \\
{[\mathrm{n} ; \% \text { of } \mathrm{n}=845]}\end{array}$} & $\begin{array}{r}\text { median (IQR) } \\
{[\mathrm{n} ; \% \text { of } \mathrm{n}=845}\end{array}$ \\
\hline $\mathrm{RV}^{\mathrm{a}}$ systolic pressure $(\mathrm{mmHg})$ & $\begin{array}{c}61(50 ; 73) \\
{[\mathrm{n}=826 ; 98 \%]}\end{array}$ & $\begin{array}{c}40(34 ; 49) \\
{[n=789 ; 93 \%]}\end{array}$ & $\begin{array}{c}-19.5(-30 ;-10) \\
{[n=778 ; 92 \%]}\end{array}$ \\
\hline $\mathrm{PA}^{\mathrm{b}}$ systolic pressure $(\mathrm{mmHg})$ & $\begin{array}{c}24(20 ; 30) \\
{[\mathrm{n}=825 ; 98 \%]}\end{array}$ & $\begin{array}{c}28(23 ; 34) \\
{[\mathrm{n}=781 ; 92 \%]}\end{array}$ & $\begin{array}{c}3(-1 ; 8) \\
{[\mathrm{n}=771 ; 91 \%]}\end{array}$ \\
\hline PA diastolic pressure $(\mathrm{mmHg})$ & $\begin{array}{c}10(8 ; 13) \\
{[n=816 ; 97 \%]}\end{array}$ & $\begin{array}{c}13(10 ; 16) \\
{[\mathrm{n}=678 ; 80 \%]}\end{array}$ & $\begin{array}{c}3(0 ; 5) \\
{[n=666 ; 79 \%]}\end{array}$ \\
\hline RV-to-PA pressure gradient ( $\mathrm{mmHg}$ ) & $\begin{array}{c}36(24 ; 47) \\
{[\mathrm{n}=821 ; 97 \%]}\end{array}$ & $\begin{array}{c}12(7 ; 17) \\
{[\mathrm{n}=777 ; 92 \%]}\end{array}$ & $\begin{array}{l}-23(-34 ;-12) \\
{[\mathrm{n}=764 ; 90 \%]}\end{array}$ \\
\hline Systemic systolic pressure (mmHg) & $\begin{array}{c}94(85 ; 106) \\
{[n=751 ; 89 \%]}\end{array}$ & $\begin{array}{l}108(95 ; 121) \\
{[n=663 ; 78 \%]}\end{array}$ & $\begin{array}{c}11(0 ; 24) \\
{[\mathrm{n}=649 ; 77 \%]}\end{array}$ \\
\hline $\mathrm{RV}$-to-systemic pressure ratio & $\begin{array}{l}0.64(0.5 ; 0.8) \\
{[\mathrm{n}=747 ; 88 \%]}\end{array}$ & $\begin{array}{l}0.37(0.3 ; 0.5) \\
{[\mathrm{n}=649 ; 77 \%]}\end{array}$ & $\begin{array}{c}-0.26(-0.4 ;-0.1 \\
{[\mathrm{n}=635 ; 75 \%]}\end{array}$ \\
\hline
\end{tabular}

RV, right ventricle; ${ }^{\mathrm{b}} \mathrm{PA}$, pulmonary artery. The $95 \%$ confidence intervals (CI) correspond to bias-corrected Significant differences in bold. 


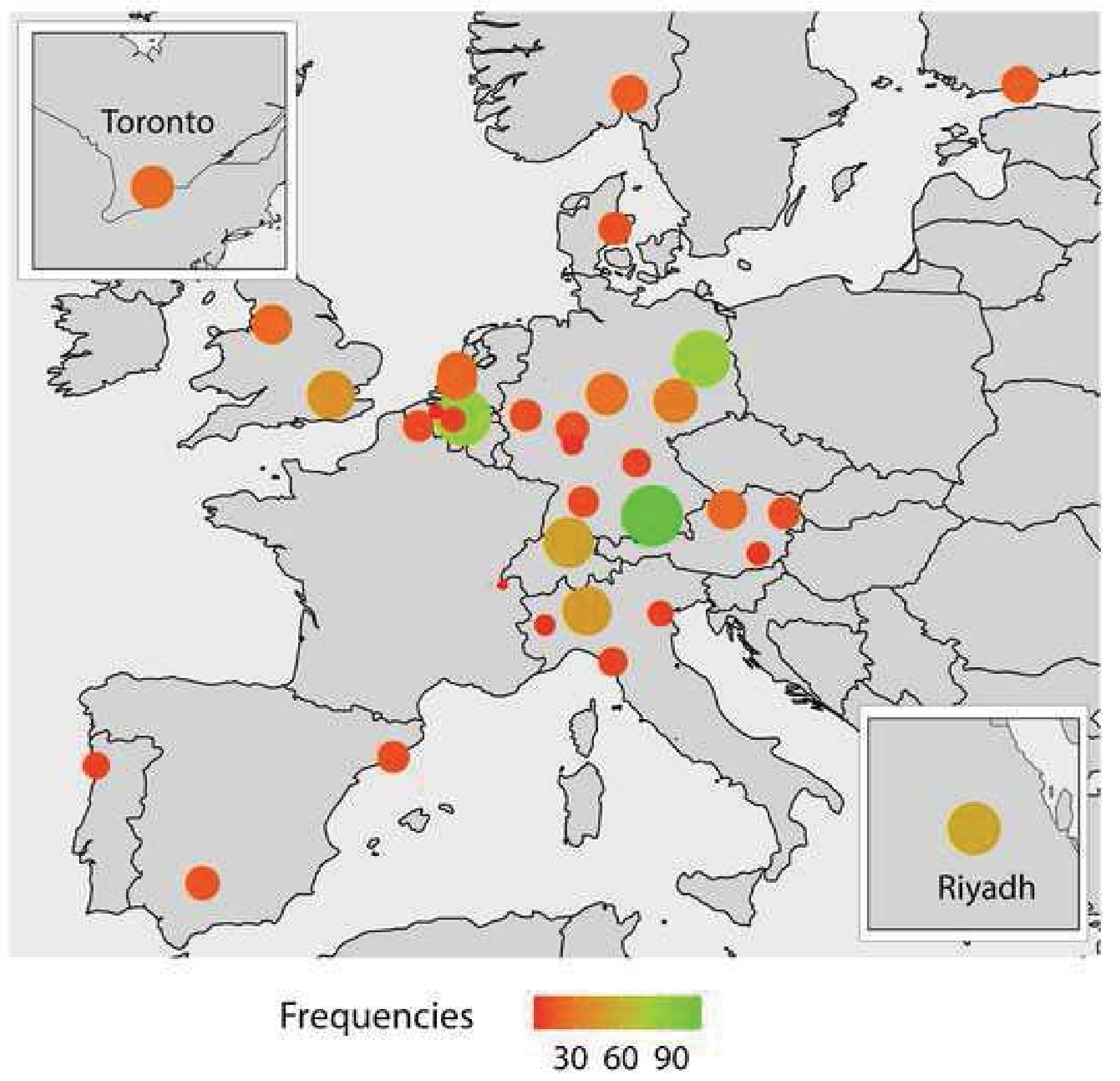


Supplemental Table 1. Procedural details, stratified by the use of pre-stenting

\begin{tabular}{|c|c|c|c|}
\hline Parameter & $\begin{array}{l}\text { Pre-Stenting - } \\
\quad(n=108)\end{array}$ & $\begin{array}{c}\text { Pre-Stenting + } \\
\quad(n=737)\end{array}$ & $P$ \\
\hline Procedure time (min), mean \pm SD & $128 \pm 46$ & $156 \pm 56$ & $<0.001$ \\
\hline Fluoroscopy time (min), mean \pm SD & $28 \pm 13$ & $33 \pm 20$ & 0.01 \\
\hline Size of Ensemble delivery system (EDS) for TPVI, $n(\%)$ & & & 0.86 \\
\hline $18-\mathrm{mm}$ & $22 / 108(20)$ & $134 / 737(18)$ & \\
\hline 20-mm & 30/108 (27) & $207 / 737(28)$ & \\
\hline 22-mm & $56 / 108(51)$ & $396 / 737(54)$ & \\
\hline Ratio of EDS size to original conduit diameter, mean \pm SD & $0.98 \pm 0.11$ & $1.05 \pm 0.18$ & $<0.001$ \\
\hline High-pressure post-dilation of TPVI, $n(\%)$ & $78 / 108(71)$ & $462 / 737(64)$ & 0.14 \\
\hline
\end{tabular}

Significant differences in bold. 
Supplemental Table 2. Stent types used for pre-stenting prior to TPVI

Number of

Stent types used for pre-stenting

implanted pre-stents

$$
(n=953)
$$

\begin{tabular}{lc}
\hline CP-Stent (NuMed), uncovered, $n(\%)$ & $241 / 953(25)$ \\
CP-Stent (NuMed), covered, $n(\%)$ & $251 / 953(26)$ \\
IntraStent MaxLD (ev3), $n(\%)$ & $237 / 953(25)$ \\
AndraStent (Andramed), $n(\%)$ & $63 / 953(7)$ \\
Other / unknown, $n(\%)$ & $161 / 953(17)$ \\
\hline
\end{tabular}


Supplemental Table 3. Details on pre-TPVI assessment of the anatomical relationship between the course of the coronary arteries and the RVOT in the five patients who suffered from coronary compression during TPVI

\begin{tabular}{lccccc}
\hline Parameter & Outcome & Diagnosis & Aortic root & Selective coronary & SCA plus balloon \\
& & & angiography & angiography (SCA) & inflation in RVOT \\
\hline Patient No. 1 & Reoperation & TOF & Yes & Yes & Yes \\
Patient No. 2 & Reoperation & s/p Ross-Operation & Yes & Yes & No \\
Patient No. 3 & Death & s/p Ross-Operation & No & No & Yes \\
Patient No. 4 & Death & TGA, VSD, PS & Yes & Yes & No \\
Patient No. 5 & Death & s/p Ross-Operation & Yes & No & No \\
\hline
\end{tabular}

RVOT, right ventricular outflow tract; TOF, Tetralogy of Fallot; TGA, transposition of the great arteries; VSD, ventricular septal defect; PS, pulmonary stenosis. 
Supplemental Table 4. Details on the events for the 'time-to-first event' analyses

Event specification and reason for event

n $(\%)$

TPVI-related reintervention, $n(\%)$

$122 / 845(14.4)$

Balloon dilation of TPVI, $n(\%)$

$72 / 122(59)$

Pulmonary restenosis

$62 / 72(86)$

TPVI infective endocarditis

$8 / 72(11)$

TPVI stent fractures

$2 / 72(3)$

Repeat TPVI as 'valve-in-valve' procedure, $n(\%)$

$50 / 122(41)$

Pulmonary restenosis

$26 / 50(52)$

TPVI infective endocarditis

$8 / 50(16)$

TPVI stent fractures

$16 / 50(32)$

TPVI-related reoperation with explantation of TPVI, $n(\%)$

$80 / 845(9.5)$

Pulmonary restenosis

$20 / 80(25)$

TPVI infective endocarditis

$53 / 80(67)$

TPVI stent fractures

$6 / 80(7)$

Chronic pericardial effusion

$1 / 80(1)$

TPVI-related death, $n(\%)$

$5 / 845(0.6)$

TPVI infective endocarditis

$5 / 5(100)$ 
Supplemental Table 5. Secondary outcomes of TPVI infective endocarditis $(n=109)$, stratified by infectious orga

\begin{tabular}{lccc}
\hline & Number of patients, $n(\%)$ & Conservative: medical therapy, $n(\%)$ & Reintervention, $n$ \\
& & & $2 / 9(22)$ \\
\hline Blood-culture negative & $9 / 109(8)$ & $1 / 9(11)$ & $11 / 100(11)$ \\
Blood-culture positive & $100 / 109(92)$ & $35 / 100(35)$ & $4 / 46(9)$ \\
\hline Staphylococcal species & $46 / 100(46)$ & $10 / 46(22)$ & $4 / 32(13)$ \\
Streptococcal species & $32 / 100(32)$ & $15 / 32(47)$ & $2 / 9(22)$ \\
HACEK organisms & $9 / 100(10)$ & $5 / 9(56)$ & $0 / 2(0)$ \\
Corynebacterium & $2 / 100(2)$ & $1 / 2(50)$ & $0 / 1(0)$ \\
Rothia dentocariosa & $1 / 100(1)$ & $0 / 1(0)$ & $0 / 1(0)$ \\
Neisseria elongate & $1 / 100(1)$ & $0 / 1(0)$ & $0 / 1(0)$ \\
Abiotrophia defective & $1 / 100(1)$ & $1 / 1(100)$ & $0 / 1(0)$ \\
Coxiella burnetii & $1 / 100(1)$ & $0 / 1(0)$ & $1 / 1(100)$ \\
Fungus & $1 / 100(1)$ & $0 / 1(0)$ & $0 / 6(0)$ \\
Other species / unknown & $6 / 100(6)$ & $3 / 6(50)$ & \\
\hline
\end{tabular}


Supplemental Table 6. Secondary outcomes of TPVI infective endocarditis $(n=109)$, divided by the subgrc according to modified Duke criteria

\begin{tabular}{|c|c|c|}
\hline & Total & possible TPVI I.E. \\
\hline Number of patients, $n(\%)$ & $109 / 109(100)$ & $41 / 109(38)$ \\
\hline \multicolumn{3}{|l|}{ Secondary outcomes } \\
\hline Conservative: medical therapy, $n(\%)$ & $36 / 109(33)$ & $19 / 41(46)$ \\
\hline Reintervention, $n(\%)$ & $13 / 109(12)$ & $5 / 41(12)$ \\
\hline Reoperation, $n(\%)$ & $51 / 109(47)$ & $15 / 41(37)$ \\
\hline Death, $n(\%)$ & $9 / 109(8)$ & $2 / 41(5)$ \\
\hline \multicolumn{3}{|l|}{ Secondary outcomes } \\
\hline Conservative: medical therapy, $n(\%)$ & $36 / 109(33)$ & $19 / 41(46)$ \\
\hline Reintervention, reoperation or death, $n(\%)$ & $73 / 109(67)$ & $22 / 41(54)$ \\
\hline \multicolumn{3}{|l|}{ Hemodynamic instability or septic syndrome } \\
\hline Present, $n(\%)$ & $28 / 109(19)$ & $3 / 41(7)$ \\
\hline Not present, $n(\%)$ & $88 / 109(81)$ & 38/41 (93) \\
\hline
\end{tabular}

Significant differences in bold. 


\section{Legends}

Supplemental Figure 1. Flow chart of patient selection with step-by-step exclusion of datasets according to predefined criteria.

Supplemental Figure 2. Number of included patients per calendar year. In this figure, two 'eras' can be identified within the MELODY Registry cohort. Between the years 2006 and 2009, the Melody ${ }^{\mathrm{TM}}$ valve was introduced to the clinical arena. During this period, there was a steep increase of included patients (i.e. first era); this increase was followed by a phase with stable number of included patients between 2010 and 2013 (i.e. more current era).

Supplemental Figure 3. The relationship between the intended size of delivered TPVI (i.e. size of Ensemble delivery system) and the original conduit diameter are displayed in violin plots, which resemble a combination of Box and distribution plots. For each intended size of delivered TPVI (i.e. size of Ensemble delivery system 18, 20 and $22 \mathrm{~mm}$, respectively) the corresponding distribution of original conduit diameters are plotted. The green boxes correspond to the Inter-quartile-range (IQR) and the bold black line to the median of the respective values. This is shown for homograft and heterograft conduits (left panel) and for bioprosthetic heart valves (right panel). The fine black line corresponds to the equation of $\mathrm{x}=\mathrm{y}$, where the intended size of the delivered TPVI would exactly correspond to the original conduit diameter. Any point that lies below the black line would correspond to a scenario, where the intended size of the delivered TPVI would be greater than the original conduit diameter (TPVI $>$ conduit). Any point that lies above the black line would correspond to a scenario, where the intended size of the delivered TPVI would be smaller than the original conduit diameter (TPVI $<$ conduit). The bold dashed red lines relate to linear regression functions that describe the average relationships between the intended size of the delivered TPVI and the original conduit diameter (in patients with homograft or heterograft conduits: $0.75^{*} \mathrm{x}+4.9, \mathrm{R}^{2}=0.13$; in patients with bioprosthetic heart valves: $0.96 * x+1.55, \mathrm{R}^{2}=0.22$ ). In case of homograft or heterograft conduits, that line illustrates that, when $20 \mathrm{~mm}$ and $22 \mathrm{~mm}$ Ensemble delivery systems were used, the intended size of delivered TPVI was chosen larger - on average - than the original conduit diameters (i.e. "oversizing”). However, in bioprosthetic heart valves, the intended size of delivered TPVI was chosen smaller - on average - than the original diameters of the bioprostheses (i.e. “undersizing”). 
Supplemental Figure 4. A, B, C: echocardiographic outcomes pre, post and 1-year after TPVI in a subset of patients in whom corresponding data was available for review (A: peak RVOT velocity $(\mathrm{m} / \mathrm{s})$, B: peak TR-jet velocity $(\mathrm{m} / \mathrm{s}), \mathrm{C}$ : pulmonary regurgitation (PR) grade). During echocardiography, the peak velocities across the right ventricular outflow tract (RVOT) and the tricuspid regurgitation (TR) jet were obtained from continuous-wave Doppler traces. Color flow Doppler mapping of the RVOT and branch pulmonary arteries was used for qualitative grading of pulmonary regurgitation (PR) from grade 0 (= none) to grade 4 (= severe). If presence of diastolic flow reversal in the main or branch pulmonary arteries was seen, PR was graded > 2. D: NYHA-class pre, post and 1-year after TPVI. The black boxes with error bars (standard deviations) correspond to the overall patient cohort, whilst the colored boxes correspond to the mean values stratified according to the primary hemodynamic lesion ( $A$ and $B)$.

Supplemental Figure 5. Details on the primary management of TPVI infective endocarditis and secondary outcomes. 
submitted datasets

$, 080 / 1,080(100 \%)$

Exclusion criteria

$n=20 / 1,080$

$n=28 / 1,060$

$\mathrm{n}=83 / 1,032$

$n=2 / 949$

$\mathrm{n}=77 / 947$

$n=21 / 870$

$n=4 / 849$
No informed consent for use of data

TPVI not performed

Implantation date prior to $29^{\text {th }}$ September 2006

(i.e. date of CE-mark approval)

Submitted dataset corresponds to repeat TPVI

No data on primary hemodynamic lesion

(i.e. indication for TPVI)

TPVI not delivered on Ensemble ${ }^{\mathrm{TM}}$ Delivery System

No data entry of 1-year follow-up in patients who did not have a TPVI-related event within 1-year (either procedural complication or during follow-up) 
B
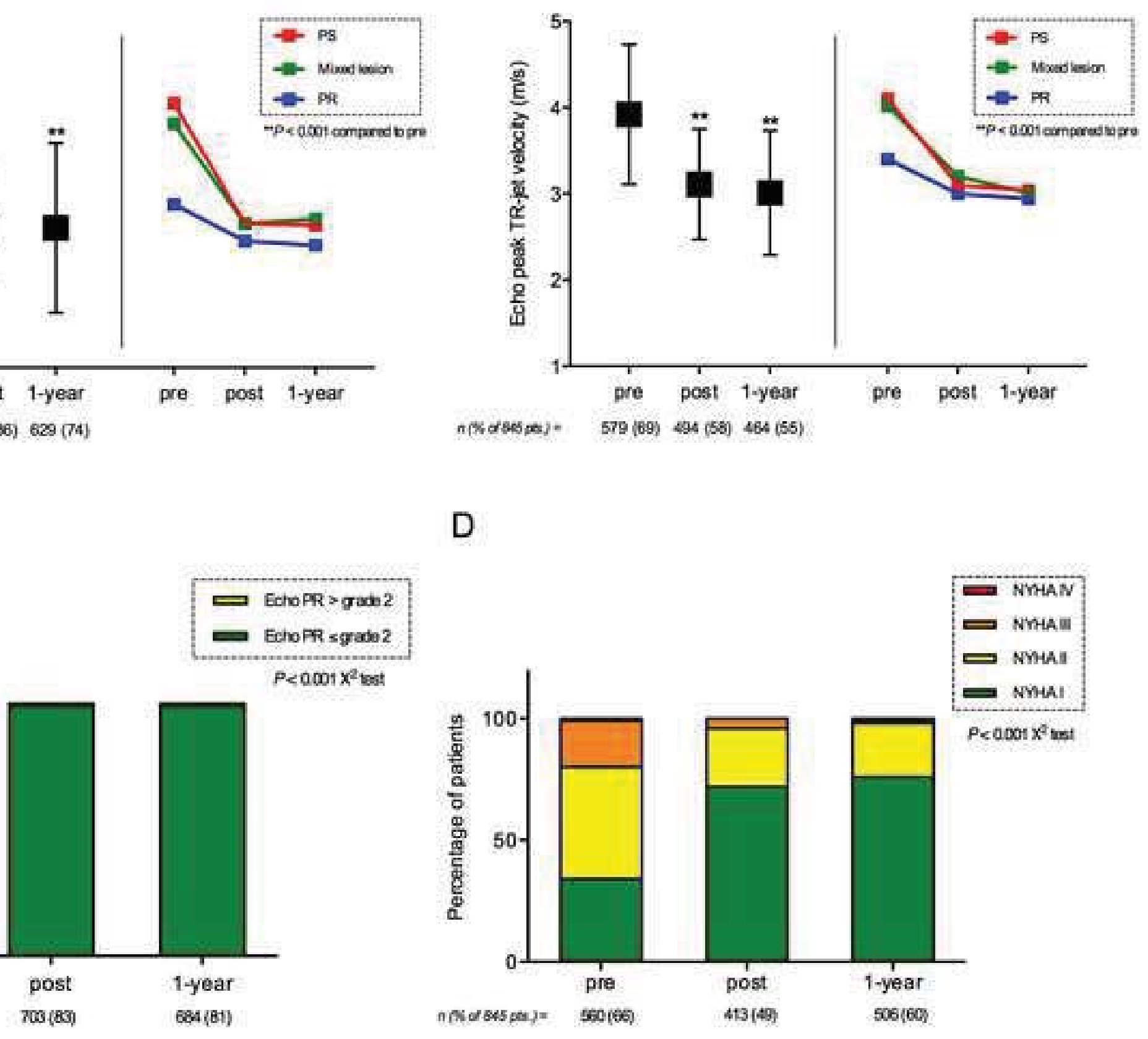


\begin{tabular}{|c|c|c|}
\hline $\begin{array}{l}\text { ative: medical therapy } \\
=41 / 109(38 \%)\end{array}$ & $n=36$ & $\begin{array}{l}\text { Conservative: medical therapy } \\
\qquad n=36 / 109(33 \%)\end{array}$ \\
\hline $\begin{array}{l}\text { Ion and medical therapy } \\
15 / 109(14 \%)\end{array}$ & $n=13 \rightarrow$ & $\begin{array}{l}\text { Reintervention and medical therapy } \\
\qquad n=13 / 109(12 \%)\end{array}$ \\
\hline $\begin{array}{l}\text { vith explantation of TPVI } \\
\text { medical therapy } \\
53 / 109(48 \%)\end{array}$ & $n=51$, & $\begin{array}{l}\text { Reoperation with explantation of TPVI and } \\
\text { medical therapy } \\
\qquad n=51 / 109(47 \%)\end{array}$ \\
\hline & $\begin{array}{l}n=2 \\
n=2 \\
n=5\end{array}$ & $\begin{array}{c}\text { Death } \\
n=9 / 109(8 \%)\end{array}$ \\
\hline
\end{tabular}


Word count

Total word count: 6,459 words

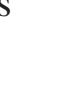

Total word count 6,459 words

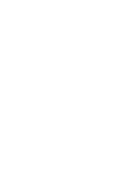

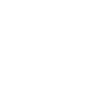

.

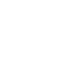

(n)

(n)

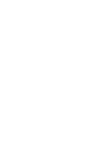

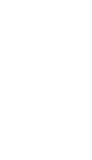

(2)

(1)

(1)

.

.

.

.
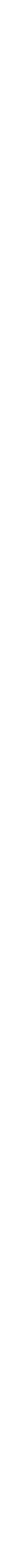
Permissions information

There are no illustrations or figures that were duplicated from previously published work.

There are no illustrations or figures that were duplicated from previously published work.

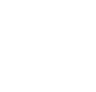

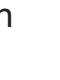

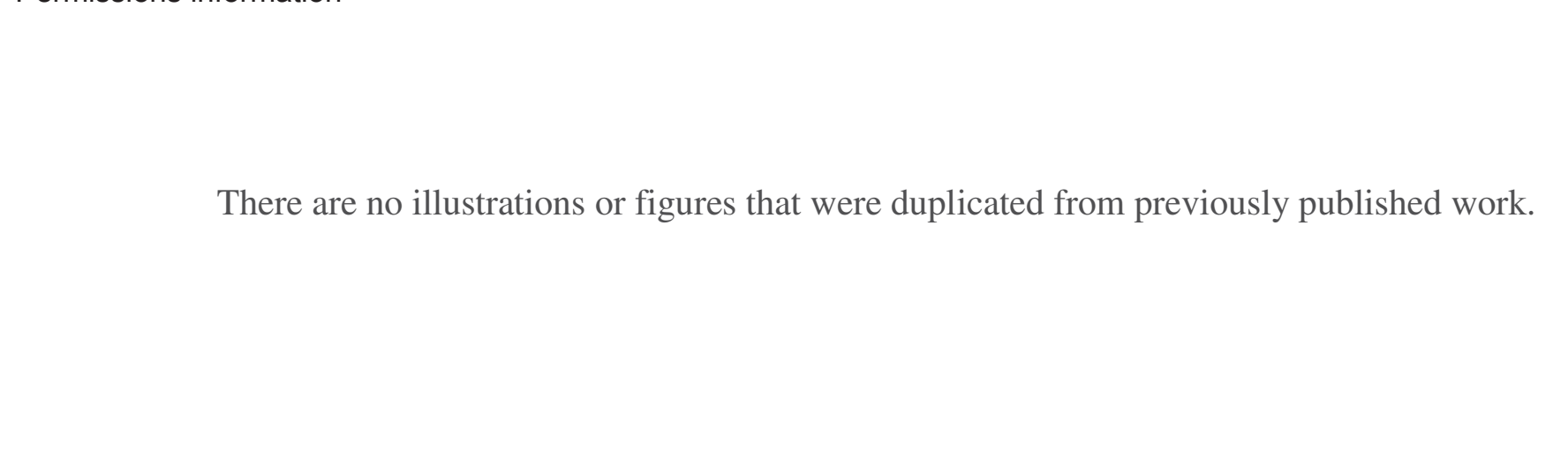

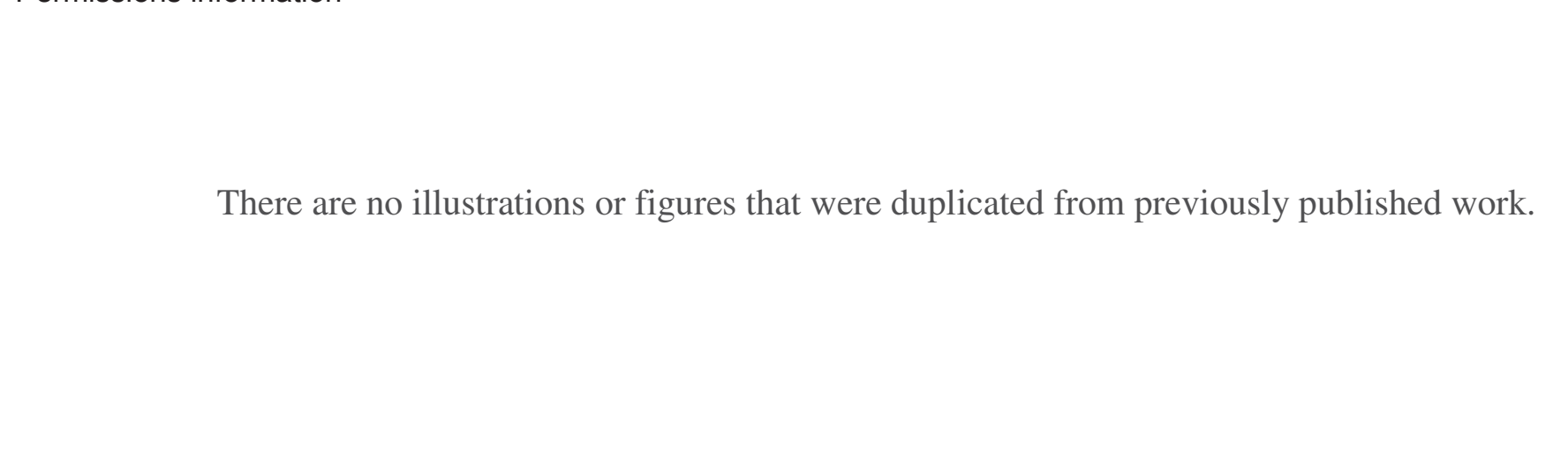

There are no illustrations or figures that were duplicated from previously published work.

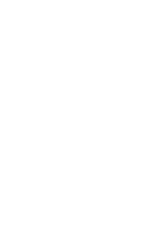

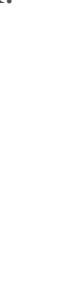

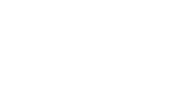

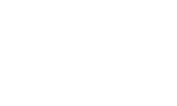

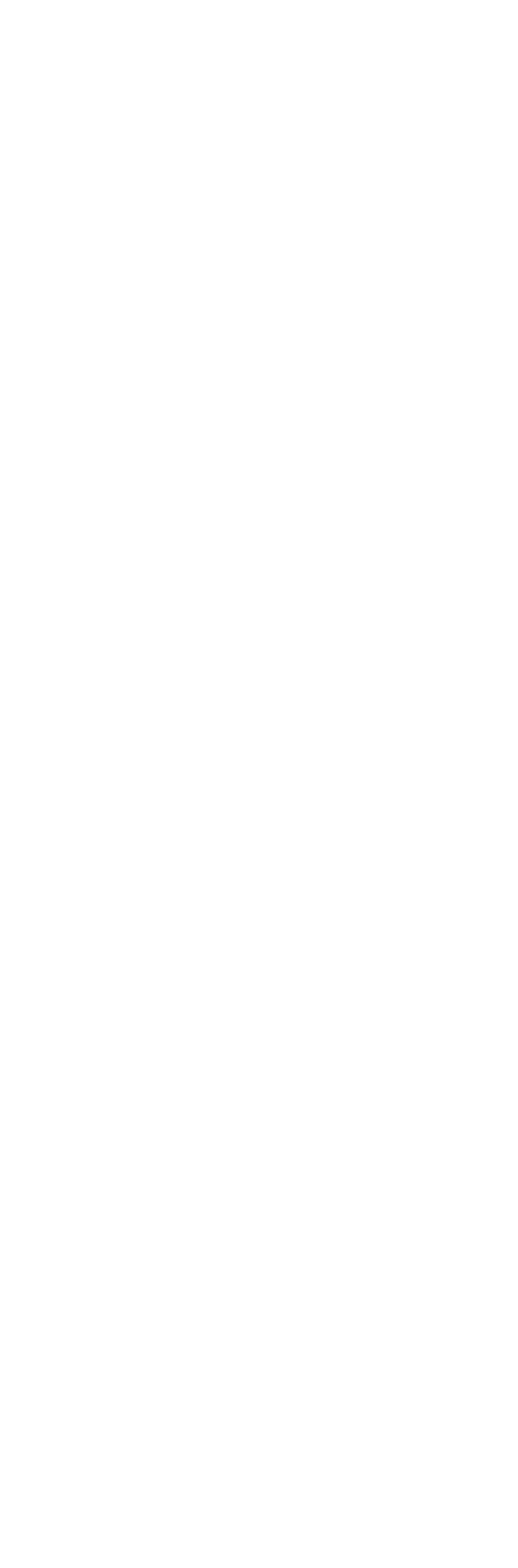




\begin{abstract}
Aims - The post-approval MELODY Registry aimed to obtain multicentre registry data after transcatheter pulmonary valve implantation (TPVI) with the Melody ${ }^{\mathrm{TM}}$ valve (Medtronic plc.) in a large-scale cohort of patients with congenital heart disease (CHD).
\end{abstract}

Methods and results - Retrospective analysis of multicentre registry data after TPVI with the Melody ${ }^{\mathrm{TM}}$ valve. 845 patients (mean age: $21.0 \pm 11.1$ years) underwent TPVI in 42 centres between 12/2006 and 09/2013 and were followed-up for a median of 5.9 years (range: 0 to 11.0 years). The composite endpoint of TPVI-related events during follow-up (i.e. death, reoperation or reintervention $>48 \mathrm{~h}$ after TPVI) showed an incidence rate of $4.2 \%$ per person per year $(95 \% \mathrm{CI}$ 3.7-4.9). TPVI infective endocarditis showed an incidence rate of $2.3 \%$ per person per year (95\% CI 1.9-2.8) and resulted in significant morbidity and in 9 deaths. In multivariable Cox proportional hazard models, the invasively measured residual RV-to-PA pressure gradient (per 5 $\mathrm{mmHg}$ ) was associated with the risk of the composite endpoint (adjusted hazard ratio: $1.21,95 \%$ CI 1.12-1.30; $P<0.0001)$ and the risk of TPVI infective endocarditis (adjusted hazard ratio: 1.19, 95\% CI 1.07-1.32; $P=0.002$ ). Major procedural complications (death, surgical or interventional treatment requirement) occurred in $0.5,1.2$, and $2.0 \%$, respectively. Acutely, the right ventricle (RV)-to-pulmonary artery (PA) pressure gradient and the percentage of patients with pulmonary regurgitation grade >2 improved significantly from 36 (interquartile range 24-47) to 12 (interquartile range $7-17) \mathrm{mmHg}$ and 47 to $1 \%$, respectively $(P<0.001$ for each).

Conclusions - The post-approval MELODY Registry confirms the efficacy of TPVI with the Melody ${ }^{\mathrm{TM}}$ valve in a large-scale cohort of CHD patients. The residual invasively measured RV-toPA pressure gradient may serve as a target for further improvement in the composite endpoint and TPVI infective endocarditis. However, TPVI infective endocarditis remains a significant concern causing significant morbidity and mortality. 